Accretion Phenomena and Related Outflows, IAU Colloquium 169

ASP Conference Series, Vol. 121, 1997

D.T. Wickramasinghe, L. Ferrario, and G.V. Bicknell, eds.

\title{
Instability, Turbulence, and Enhanced Transport in Accretion Disks
}

\author{
Steven A. Balbus and John F. Hawley \\ VITA, Astronomy Department, University of Virginia, Charlottesville \\ $V A 22903$
}

\begin{abstract}
The nature of MHD and hydrodynamical turbulence in accretion disks is discussed. Comparison is made with planar Couette flow, a classical system prone to nonlinear shear instability resulting in enhanced turbulent transport. Both Keplerian and non-Keplerian hydrodynamical disks are studied, and it is found that only constant angular momentum disks are unstable to nonlinear disturbances and develop enhanced turbulent transport. Convective instabilities do not lead to enhanced turbulent transport. Hydrodynamical Keplerian disks are quite stable to nonlinear disturbances. Several lines of argument are presented which all lead to this conclusion, but the key to disk turbulence is the interaction between the stress tensor and the mean flow gradients. The nature of this coupling is found to determine completely the stability properties of disks (hydrodynamics and magnetic), and the nature of turbulent transport. The weak field MHD instability, which is of great astrophysical importance, displays the same type of stress tensor - mean flow coupling that all classical local shear instabilities exhibit. Hydrodynamical Keplerian disks, on the other hand, do not. Accretion disk turbulence is MHD turbulence.
\end{abstract}

\section{Introduction}

The time when one might justifiably characterize the origin of accretion disk turbulence as "mysterious" is now long past. The presence of disk turbulence is no more fundamentally mysterious than that of the convective turbulence which develops in the outer layers of low mass stars. Like its Schwarzschild counterpart, the disk stability criterion is simple to obtain and easy to state: weakly magnetized disks (i.e., those with subthermal fields) are dynamically unstable if the angular velocity decreases outwards (Balbus \& Hawley 1991). As many contributions to this conference proceeding show, there is certainly no shortage of mysteries confronting investigators of accretion disks. But making up a form for the stress tensor is no longer a prerequisite for progress.

Secure in the knowledge that turbulent stresses in disks are true dynamical entities, we can be somewhat more ambitious with respect to the type of questions we might profitably ask. For example, in high Reynolds number classical shear layer (planar Couette) flow, the nature of the interaction between the turbulent eddies and the mean flow is well-understood (e.g., Tennekes \& Lumley 1972). Vortices in the flow are caught up in, and stretched by the shear. Since 
the vorticity of a fluid element tends to be conserved, a stretched vortex rotates more rapidly. The fluctuations in the cross-stream and streamwise velocities are correlated by this effect, and it is the correlation in these velocity components that gives rise to enhanced turbulent Reynolds transport.

The coupling between the turbulent stress and the mean flow-in this example, the background velocity shear-is also the energy source that maintains turbulence against its own dissipative tendencies. It is not an accident that enhanced transport is present in planar Couette flow only when the the source of free energy for the turbulence is the shear. It is the process of extracting this energy that forces fluctuation velocities to become correlated. Notice that it is not simply the existence of turbulent flow that enhances viscous transport, as is often taken for granted. There must be some reason for turbulent velocities to be correlated.

In this review, we aim to treat the problem of accretion disk turbulence at a level comparable to that of shear layers. But the differences between differential rotation and Cartesian shear flow are profound, and that conditions for nonlinear stability and enhanced transport in the two systems are by no means analogous. We can in fact make an even stronger statement: hydrodynamical turbulence cannot, as a matter of principle, result in enhanced transport in Keplerian disks. While such systems can transport angular momentum via large scale waves, only MHD turbulence can achieve what we know to be happening in shear layer turbulence: the extraction of free energy from shear by the correlated components of a turbulent stress tensor.

\section{Hydrodynamical Disks}

\subsection{Formal Considerations}

Let $\mathbf{u}=\mathbf{v}-R \Omega \hat{\phi}$ be the difference between the velocity $\mathbf{v}$ and the circular velocity $R \Omega$ in a disk. (We use standard $R, \phi, z$ cylindrical coordinates. $\Omega$ is the angular velocity.) In the absence of a magnetic field, only one quantity is of consequence to enhancing the transport: $\left\langle\rho u_{R} u_{\phi}\right\rangle$, where the angle brackets denote a suitably defined average (the correlation average is supposed to be smoothly varying in time and space), and $\rho$ is the density. Physically, this correlation represents the nonadvective piece of the angular momentum transport. The net radial component of the angular momentum flux $\mathcal{F}_{\mathcal{J}}$ is:

$$
\mathcal{F}_{\mathcal{J}}=R\left\langle\rho\left(u_{\phi}+R \Omega\right) u_{R}\right\rangle=R^{2} \Omega\left\langle\rho u_{R}\right\rangle+R\left\langle\rho u_{R} u_{\phi}\right\rangle
$$

What makes matters complicated is that $\left\langle\rho u_{R} u_{\phi}\right\rangle$ is also the "tap" into the mean flow which siphons energy and angular momentum into the turbulent velocity fluctuations.

To see more fully the implications of this dual role, consider the equations of motion for the $u$ velocities,

$$
\begin{aligned}
& \left(\frac{\partial}{\partial t}+\Omega \frac{\partial}{\partial \phi}+\mathbf{u} \cdot \nabla\right) u_{R}-2 \Omega u_{\phi}=-\frac{1}{\rho} \frac{\partial P}{\partial R}+\nu \nabla^{2} u_{R} \\
& \left(\frac{\partial}{\partial t}+\Omega \frac{\partial}{\partial \phi}+\mathbf{u} \cdot \nabla\right) u_{\phi}+\frac{\kappa^{2}}{2 \Omega} u_{R}=-\frac{1}{\rho R} \frac{\partial P}{\partial \phi}+\nu \nabla^{2} u_{\phi}
\end{aligned}
$$


which follow directly from the standard equations of motion under the assumption that $u \ll R \Omega$. Here,

$$
\kappa^{2}=\frac{2 \Omega}{R} \frac{d R^{2} \Omega}{d R}
$$

is the usual epicyclic frequency, and we have retained what will prove to be the only important term proportional to the (true) kinematic viscosity $\nu$. If we multiply equation (2) by $\rho u_{R}$ and equation (3) by $\rho u_{\phi}$, regroup, and average (including the full $2 \pi$ azimuth therein), we may write the results as

$$
\begin{gathered}
\frac{\partial}{\partial t}\left\langle\frac{1}{2} \rho u_{R}^{2}\right\rangle+\left\langle\nabla \cdot \mathcal{F}_{R}\right\rangle=2 \Omega\left\langle\rho u_{R} u_{\phi}\right\rangle+\left\langle P \frac{\partial u_{R}}{\partial R}\right\rangle+\nu\left\langle\rho\left|\nabla u_{R}\right|^{2}\right\rangle \\
\frac{\partial}{\partial t}\left\langle\frac{1}{2} \rho u_{\phi}^{2}\right\rangle+\left\langle\nabla \cdot \mathcal{F}_{\phi}\right\rangle=-\frac{\kappa^{2}}{2 \Omega}\left\langle\rho u_{R} u_{\phi}\right\rangle+\left\langle\frac{P}{R} \frac{\partial u_{\phi}}{\partial \phi}\right\rangle+\nu\left\langle\rho\left|\nabla u_{\phi}\right|^{2}\right\rangle
\end{gathered}
$$

where $\mathcal{F}_{\mathcal{R}}$ and $\mathcal{F}_{\phi}$ are fluxes whose precise form does not concern us. What does concern us is that the turbulent source terms, $2 \Omega\left\langle\rho u_{R} u_{\phi}\right\rangle$ and $-\left(\kappa^{2} / 2 \Omega\right)\left\langle\rho u_{R} u_{\phi}\right\rangle$ cannot both be "source" terms: they have opposite signs! This is of course the profoundly stabilizing effect of epicyclic oscillations at work. (We note in passing the mathematically trivial but physically important point that Coriolis forces couple to the turbulence precisely as does a velocity shear. Although they cannot affect the energy transfer to the turbulence, Coriolis forces most certainly can affect the angular momentum transfer.) We must have some source term on the right hand sides to balance the viscous losses. In a shear layer, the source terms are very different. There would be no Coriolis term in the analogue of equation (4), and the $\kappa^{2}$ term in equation (5) would be replaced with a velocity gradient. The ensuing nonlinear instability, fed by vortex stretching, proceeds apace. A postive correlation in cross-stream and stream-wise velocities in this case would produce no dynamical counter-tendencies, no inclination to grow one component at the expense of the other.

But what of the pressure terms? We have said little about them thus far. Could they not act as a source terms for the turbulence, making our focus on $\left\langle\rho u_{R} u_{\phi}\right\rangle$ too narrow? Indeed, under some circumstances the pressure term in equation (5) is the dominant source. This happens in regions of the solar convection zone, where angular momentum is pumped uphill against its gradient (hardly an enhanced viscosity!), and it happens also in turbulence generated by self-gravity in disks (Gammie 1996). For this term to dominate, however, requires a good correlation between pressure fluctuations and velocity gradient fluctuations. But if anything like a classic turbulent cascade is present, their power spectra are very different: pressure fluctuations are dominated by the largest scales, velocity gradients (in essence vorticity) by the smallest. It is difficult to overcome the competing $\left\langle\rho u_{R} u_{\phi}\right\rangle$ correlation term under these circumstances, since the velocities have their respective power spectra both concentrated on the largest scales. What therefore tends to happen when pressure torques dominate angular momentum transport, is that a global pattern emerges, and the velocity gradient power spectrum forms a good correlation with the large scale pressure fluctuations (e.g., Spruit 1987, Hawley 1991). The result: in the sun, a global flux pattern that circulates, but does not extract, angular momentum; in galaxies, spiral structure. 
If, however, what is desired is an "eddy viscosity" $\alpha$ disk model along the lines of a turbulent shear layer, options are extremely limited. The hydrodynamical behavior of an accretion disk is completely different from that of a shear layer, a difference which stems from the presence of epicyclic oscillations as opposed to simple shear. If our reasoning is correct, then in a $\kappa^{2}=0$ (constant specific angular momentum) disk, we ought to recover the behavior of a shear layer. This is apparent from the form of equations (4) and (5), which take on the character of a shear layer: one equation with a velocity correlation source, the other without. As discussed more fully in the companion article (Hawley \& Balbus 1996), numerical simulations show precisely this effect. Nonlinear instability is recovered for shear layers and $\kappa^{2}=0$ disks, but for no other cases.

\subsection{Numerical Simulations}

The failure to uncover any nonlinear instabilities in standard Keplerian disks is a result of some significance, for a standard orthodoxy of many years is that shearing disks should behave like planar Couette flow (e.g. Zahn 1987). The usual argument that such instabilities cannot be uncovered numerically because the required critical Reynolds $\mathcal{R}$ number is too high, does not wash. Nonlinear shear instabilities are readily recovered where they ought to be. Furthermore, their presence or absence has little to do with grid resolution, and everything to do with physical flow dynamics.

We have undertaken the numerical study of a series of local disk models based on equations (2) and (3). Details of the implementation may be found in Balbus, Hawley, \& Stone (1996). We parameterized the unperturbed circular velocity by $\Omega \sim R^{-q}$, so that $q=2$ corresponds to a constant angular momentum disk, and $q=3 / 2$ corresponds to a Keplerian disk. If $q>2$, the disk should be linearly unstable by the Rayleigh criterion. The results are shown in figure [1], which plots the growth of the fluctuation kinetic energy as a function of time.

When $q=2.1$ (Rayleigh Unstable), the flow is indeed found to be linearly unstable at the correct growth rate. Note that this simple result is itself an indication of a large effective $\mathcal{R}$ : G.I. Taylor's original narrow gap Couette flow calculation predicted linear instability only for $\mathcal{R}>10^{3}$ or so (Drazin \& Reid 1981).

When $q=2$ (Constant $L$ ), the prediction is born out that nonlinear instability is present. Furthermore, when a direct simulation of a pure shear layer is carried through, the results are statistically indistinguishable from the $q=2$ run.

The most telling finding is the $q=1.95$ run. With this slight change in exponent, the only change in the fluid dynamics is to introduce a small sink term (if $\left\langle\rho u_{R} u_{\phi}\right\rangle>0$ ) in the angular momentum equation (5). But the qualitative results are dramatically different. Now, neither outward nor inward transport can lead to the active reenforcement of velocity fluctuations seen in the $q=2.1$ run, nor to the nonlinear growth of the $q=2$ run. With this tiny change in the circular velocity index, and no other change in the numerics, the disk has become completely stable. All trace of nonlinear instability has disappeared. Continued decline in $q$ results in nothing qualitatively new; by the time the Keplerian $q=3 / 2$ value is reached, stability has long ruled. Even direct "stirring" of the disk at this point is unable to generate the slightest hint of outward transport 


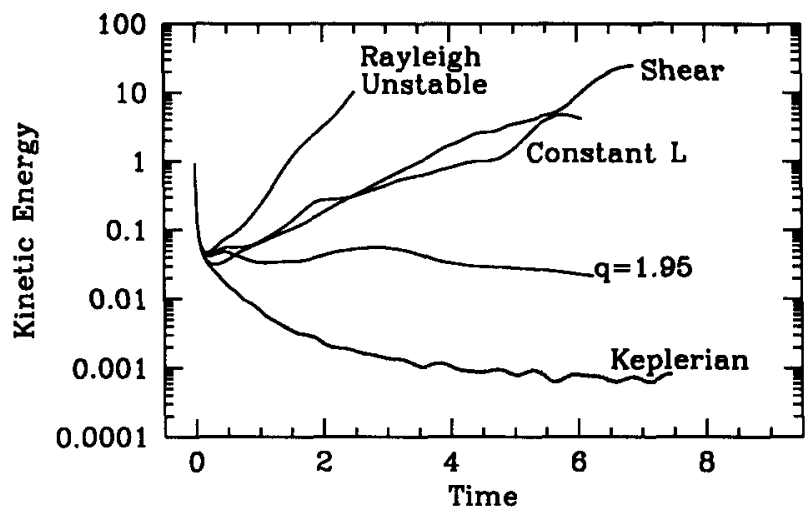

Figure 1. Evolution of kinetic energy for disks described by power law angular velocities of the form $\Omega \sim r^{-q}$. The Rayleigh Unstable curve corresponds to $q=2.1$, the Constant $L$ curve to $q=2$, and the Keplerian curve to $q=3 / 2$. A shear layer curve, modeled as a Keplerian flow without local Coriolis or tidal forces, is also shown. Time is reckoned in orbits.

(Hawley \& Balbus 1996). In summary, prospects for generating an enhanced anomalous viscosity by hydrodynamical processes, particularly those involving the old warhorses of convection (Stone \& Balbus 1996) and nonlinear shear instabilities, have never looked more bleak.

Everything changes the moment a magnetic field is added to the disk.

\section{Hydromagnetic Disks}

\subsection{Formal Considerations}

The magnetic counterparts to equations (2) and (3) are

$$
\begin{aligned}
& \left(\frac{\partial}{\partial t}+\Omega \frac{\partial}{\partial \phi}+\mathbf{u} \cdot \nabla\right) u_{R}-2 \Omega u_{\phi}=-\frac{1}{\rho} \frac{\partial P_{t o t}}{\partial R}++\frac{\mathbf{B}}{4 \pi \rho} \cdot \nabla B_{R}+\nu \nabla^{2} u_{R} \\
& \left(\frac{\partial}{\partial t}+\Omega \frac{\partial}{\partial \phi}+\mathbf{u} \cdot \nabla\right) u_{\phi}+\frac{\kappa^{2}}{2 \Omega} u_{R}=-\frac{1}{\rho R} \frac{\partial P_{t o t}}{\partial \phi}+\frac{\mathbf{B}}{4 \pi \rho} \cdot \nabla B_{\phi}+\nu \nabla^{2} u_{\phi}
\end{aligned}
$$

where $P_{\text {tot }}=P+B^{2} / 8 \pi$ is the total pressure including the magnetic contribution. We assume that the Alfvén speed

$$
\mathbf{u}_{\mathbf{A}}=\frac{\mathbf{B}}{\sqrt{4 \pi \rho}}
$$

satisfies $u_{A} \ll R \Omega$, as does the fluctuating $u$ velocity. This justifies the neglect of the $B_{\phi}^{2} /(4 \pi \rho R)$ term in equation (6), and the $B_{\phi} B_{R} /(4 \pi \rho R)$ term in equation (7). In addition, we need the components of the induction equation

$$
\frac{\partial \mathbf{B}}{\partial t}=\nabla \times(\mathbf{v} \times \mathbf{B})+\eta \nabla^{2} \mathbf{B}
$$


written in term of the $u$ velocities. Here, $\eta$ is of course the microscopic resistivity. In component form we have

$$
\begin{gathered}
\frac{D B_{R}}{D t}=-B_{R} \nabla \cdot \mathbf{u}+\mathbf{B} \cdot \nabla u_{R}+\eta \nabla^{2} B_{R} \\
\frac{D B_{\phi}}{D t}-B_{R} \frac{d \Omega}{d \ln R}=-B_{\phi} \nabla \cdot \mathbf{u}+\mathbf{B} \cdot \nabla u_{\phi}+\eta \nabla^{2} B_{\phi}
\end{gathered}
$$

where

$$
\frac{D}{d t} \equiv \frac{\partial}{\partial t}+\Omega \frac{\partial}{\partial \phi}+\mathbf{u} \cdot \nabla
$$

As in our treatment of the hydrodynamics, we recast equations (6)-(9) into energy-like forms. After some integration by parts, we arrive at

$$
\begin{gathered}
\left.\frac{\partial}{\partial t}_{e q}\left(\frac{1}{2} \rho u_{R}^{2}\right)+\nabla \cdot\right]=2 \Omega \rho u_{R} u_{\phi}-u_{R} \frac{\partial P_{t o t}}{\partial R}-u_{A R} \mathbf{u}_{\mathbf{A}} \cdot \nabla u_{R}-\nu\left|\nabla u_{R}\right|^{2} \\
\frac{\partial}{\partial t}_{e q}\left(\frac{1}{2} \rho u_{\phi}^{2}\right)+\nabla \cdot\left[=-\frac{\kappa^{2}}{2 \Omega} \rho u_{R} u_{\phi}-\frac{u_{\phi}}{R} \frac{\partial P_{t o t}}{\partial \phi}-u_{A \phi} \mathbf{u} \cdot \nabla u_{\phi}-\nu\left|\nabla u_{\phi}\right|^{2}\right. \\
\frac{\partial}{\partial t}_{e q}\left(\frac{1}{2} \rho u_{A R}^{2}\right)+\nabla \cdot\left[=-\frac{1}{2} \rho u_{A R}^{2} \nabla \cdot \mathbf{u}+u_{A R} \mathbf{u}_{\mathbf{A}} \cdot \nabla u_{R}-\eta\left|\nabla B_{R}\right|^{2}\right. \\
\left.{\frac{\partial}{\partial t_{e q}}}_{e q}\left(\frac{1}{2} \rho u_{A \phi}^{2}\right)+\nabla \cdot\right]-\frac{d \Omega}{d R} \rho u_{A R} u_{A \phi}=-\frac{1}{2} \rho u_{A \phi}^{2} \nabla \cdot \mathbf{u}+u_{A \phi} \mathbf{u}_{\mathbf{A}} \cdot \nabla u_{\phi}-\eta\left|\nabla B_{\phi}\right|^{2}
\end{gathered}
$$

where

$$
\frac{\partial}{\partial t}_{e q}=\frac{\partial}{\partial t}+\Omega \frac{\partial}{\partial \phi}
$$

is the time derivative along circular orbits. The notation [] denotes a generic flux term, which being part of a pure divergence, is neither a source nor a sink for the fluctuations. (Once again, its form is unimportant for present purposes.) But the terms proportional to velocity gradients taken along magnetic field lines (i.e., $\left.\mathbf{u}_{\mathbf{A}} \cdot \nabla\right)$, which appear throughout these equations, are not expressible as pure divergences. Their role is to shuffle energy back and forth between the kinetic and magnetic fluctuations; they appear in both the dynamical and induction equations, but with opposite signs. By combining the two radial equations and the two azimuthal equations, then averaging, we obtain a pair of equations of interest:

$$
\begin{aligned}
\frac{\partial}{\partial t}\left\langle\frac{1}{2} \rho\left(u_{R}^{2}+u_{A R}^{2}\right)\right\rangle+\nabla \cdot\langle\rangle= & 2 \Omega\left\langle\rho u_{R} u_{\phi}\right\rangle-\left\langle u_{R} \frac{\partial P_{t o t}}{\partial R}\right\rangle-\frac{1}{2} \rho u_{A R}^{2} \nabla \cdot \mathbf{u} \\
& -\left\langle\nu\left|\nabla u_{R}\right|^{2}\right\rangle-\left\langle\eta\left|\nabla B_{R}\right|^{2}\right\rangle \\
\frac{\partial}{\partial t}\left\langle\frac{1}{2} \rho\left(u_{\phi}^{2}+u_{A \phi}^{2}\right)\right\rangle+\nabla \cdot\langle\rangle= & -2 \Omega\left\langle\rho u_{R} u_{\phi}\right\rangle-\frac{d \Omega}{d \ln R}\left\langle\rho\left(u_{R} u_{\phi}-u_{A R} u_{A \phi}\right)\right\rangle \\
& -\left\langle u_{\phi} \frac{\partial P_{t o t}}{\partial R}\right\rangle-\frac{1}{2} \rho u_{A \phi}^{2} \nabla \cdot \mathbf{u} \\
& -\left\langle\nu\left|\nabla u_{\phi}\right|^{2}\right\rangle-\left\langle\eta\left|\nabla B_{\phi}\right|^{2}\right\rangle
\end{aligned}
$$


Once again, we have suppressed pure divergence terms.

The reader should not be put off by the unwieldy character of these equations, the analogues of equations (4) and (5). The contribution of the $\nabla \cdot \mathbf{u}$ terms is generally unimportant since it relies on significant density fluctuations; the key new feature is the new coupling to the stress tensor that appears in equation (15). The angular momentum gradient (in the form of $\kappa^{2}$ ) is no longer the effective turbulent source term, a role for which it was most unsuited. The presence of the magnetic field introduces angular velocity coupling to the stress tensor, and this is what gives us the possibility of sustained outward turbulent transport. The energy equation (including vertical motions if needed) is simply

$$
\begin{aligned}
\frac{\partial}{\partial t}\left\langle\frac{1}{2} \rho\left(u^{2}+u_{A}^{2}\right)\right\rangle+\nabla \cdot\langle\rangle= & -\frac{d \Omega}{d \ln R}\left\langle\rho\left(u_{R} u_{\phi}-u_{A R} u_{A \phi}\right)\right\rangle+\langle P \nabla \cdot \mathbf{u}\rangle \\
& -\sum_{i}\left\langle\nu\left|\nabla u_{i}\right|^{2}+\eta\left|\nabla B_{i}\right|^{2}\right\rangle
\end{aligned}
$$

where the final sum over components $i$ represents net viscous and resistive losses. The $P \nabla \cdot \mathbf{u}$ term, where $P$ is now simply the gas pressure, is the only thermodynamic link to possible heating sources. It ought not to be casually dropped (as is sometimes done) on the justification that the motions are nearly incompressible.

Even though equation (15) contains a stabilizing Coriolis term, it loses compared with the magnetically-aided angular velocity couple. This can be shown directly for linear fluctuations, and numerical simulations show that this carries over into the nonlinear regime as well. Thus, the effect of a magnetic field is to recreate the key destabilizing dynamical features of shear layers and Rayleigh unstable disks. First, there is a return to shear as the dynamical source of turbulent (angular) momentum and energy fluctuations. Second, since. the Coriolis term appears as a dynamical source in equation (14), the instability is a linear one. A positive $u_{R} u_{\phi}$ correlation results in an active feedback loop to cause an exponential runaway, a feature in common with the Rayleigh instability.

The detailed physical explanation of the magnetic instability has been presented elsewhere (Balbus \& Hawley 1992), and we will not repeat it here. Suffice it to note the the Lagrangian fluid elements of a magnetized disk satisfy the same local equations as do orbiting point masses connected by a spring. The instability arises when one mass loses angular momentum, the other gains, and the subsequent separation of the masses stretches the spring and increases the tension torques yet further. It is interesting and not coincidental to note that the direction of fluid displacement that grows the most rapidly is at an angle of $45^{\circ}$ relative to the disk shear (Balbus \& Hawley 1992), which is also the direction that the vorticity points relative to the shear in those eddies extracting the maximum free energy from the flow (Tennekes \& Lumley 1972).

\section{Discussion}

Short of a controled laboratory experiment, the final arbiter of stability, particularly nonlinear stability, should be a numerical simulation. This has been the approached adopted by the authors for some time (Hawley \& Balbus 1996). Nevertheless, the results we have found have an internal physical sensibility to 
them which greatly enhances their plausibility. In this section, we will put forth some of these arguments.

The first point is that epicyclic motions are an anathema to angular momentum in general, and to disk turbulence in particular. In every mechanism (turbulent or otherwise) where simulations have shown outward angular momentum transport, epicyclic motion is in some way compromised. In shear layers and $\kappa^{2}=0$ disks there are no epicycles; in the magnetorotational instability and Rayleigh instability, epicyclic motion is destroyed; and when spiral density waves are generated, they are 'swing amplified' precisely because the $\kappa^{2}$ effective spring constant is dramatically softened (Toomre 1981). A nonlinear shear instability in a Keplerian disk would be utterly at odds with this trend.

Next, consider the energetics of turbulent mixing. In the case of a shear layer, at fixed (streamwise) momentum the kinetic energy of the flow is minimized when the stream velocity is constant; for a differentially rotating disk, energy is minimized at fixed total angular momentum when the angular velocity is constant. In every case where a linear or nonlinear instability has been found to lead to local turbulence, this is directly reflected in the appropriate momentum equation coupling. In a shear layer, the turbulent coupling term has the form

$$
-V^{\prime}(x)\left\langle\rho u_{x} u_{y}\right\rangle
$$

where $V^{\prime}(x)$ is the large scale cross-stream velocity gradient. An outwardly decreasing velocity gradient couples to an outward transport in the correlation tensor as the fluid tries to lower its energy by redistributing momentum. The case of a $\kappa^{2}=0$ disk has its coupling given by

$$
2 \Omega\left\langle\rho u_{R} u_{\phi}\right\rangle \equiv-\frac{d \Omega}{d \ln R}\left\langle\rho u_{R} u_{\phi}\right\rangle
$$

but for the $R$, not the $\phi$, velocity (cf eq. [4]). Here it is an outwardly decreasing angular velocity that triggers outward transport, as the disk locally tries to lower its energy by moving angular momentum outward and heading toward a state of uniform angular velocity. Next, the dominant magnetic disk couple in the $\phi$ equation (15)

$$
-\frac{d \Omega}{d \ln r}\left\langle\rho\left(u_{R} u_{\phi}-u_{A R} u_{A \phi}\right)\right\rangle
$$

which attempts to do the same energy-lowering-by-mixing in a magnetized disk. The $2 \Omega$ term appearing in the $R$ equation (14) aids in destabilization when $d \Omega / d R<0$; if the angular velocity increases outward, there is no such assistance. Finally, Rayleigh unstable disks follow the same pattern, because the angular momentum gradient and the angular velocity gradient both have the same signs. In this case, the disk is able to head in the direction of lower energy by mixing angular momentum.

In none of the cases where nonlinear stability is numerically inferred do we see a destabilizing couple associated with energy transport. Indeed, the most salient feature is the extreme numerical sensitivity to the presence of precisely this sort of couple; a trigger set off by the smallest of magnetic fields, or a tiny adverse angular momentum gradient. Hydrodynamical Keplerian disks are not remotely close to this unstable regime. 
Finally, let us take more seriously the 'isomorphism' between shear layers and $\kappa^{2}=0$ hydrodynamical disks. What this is telling us is that a shear layer may be approached asymptotically as the continuous limit of a family of disk solutions. In particular, focus on the inertial modes of the disk, whose characteristic frequency $\kappa^{2}$ derives from the restoring Coriolis force of the epicycles. In the limit $\kappa^{2} \rightarrow 0$ these modes do not disappear, they become zero frequency modes. The response frequency in general is simply a measure of the net restoring force on a fluid element; when it vanishes it means that the net sum of the linear restoring forces is zero. This is the reason that nonlinear forces become important and push the system into instability-there is nothing in the linear domain for them to compete with. Thus, instability is not simply a matter of noting that differential rotation is present in disks so the flow must be like a shear layer. It is the very specific circumstances of the presence of these zero frequency modes that enables a $\kappa^{2}=0$ disk, and thus a shear layer, to become dominated by nonlinear dynamics. (In passing, it is of interest to note that the local Alfvén and slow modes of a magnetized disk become zero frequenecy modes in the limit of vanishing field. It is the slow mode that becomes unstable in a weakly magnetized disk.) In an unmagnetized disk with a healthy positive value of $\kappa^{2}$, there are no zero frequency modes to be acted upon. Such disks are found to be very stable; any putative local nonlinear instability would be extremely difficult to place in any sensible context.

In conclusion, we believe that many features of the onset of turbulence in disks are now well understood and that the body of laboratory (see Balbus, Hawley \& Stone 1996 for a review) and numerical simulation experiments point to a consistent physical picture. Turbulence in Keplerian accretion disks resulting in enhanced viscous transport must be MHD turbulence. We are still very ignorant, of course, when it comes to the actual dynamics of turbulence, and we are likely to remain that way for some time to come. But the study of accretion disk turbulence can now be approached at a level of detail comparable to what is possible in stellar convection studies (Brummell, Cattaneo, \& Toomre 1995). Little has been done in the realm of the phenomenology of MHD turbulence, but we are at least seeing a start (e.g., Tout 1996). The subject is immature, and still of a form amenable to simple, key ideas. It is not obvious that everything that is obvious has already been done.

Acknowledgments. This work is supported by NASA grants NAG 5-3058, NAGW-4431, and NSF grant AST-9423187.

\section{References}

Balbus, S. A., \& Hawley, J. F. 1991, ApJ, 376, 214

Balbus, S. A., \& Hawley, J. F. 1992, ApJ, 392, 662

Balbus, S. A., \& Hawley, J. F., \& Stone, J. M. 1996, ApJ, 467, 76

Brummell, N., Cattaneo, F., \& Toomre, J. 1995, Science, 269, 1370

Drazin, P. G., \& Reid, W. H. 1981, Hydrodynamic Stability (Cambridge: Cambridge University Press)

Gammie, C. F. 1996, these proceedings

Hawley, J. F. 1991, ApJ, 381, 496 
Hawley, J. F., \& Balbus, S. A. 1996, these proceedings

Spruit, H. 1987, A\&A, 184, 173

Stone, J. M., \& Balbus, S. A. 1996, ApJ, 464, 364

Tennekes, H., \& Lumley, J. L. 1972, A First Course in Turbulence (Cambridge: MIT Press)

Toomre, A. 1981, in The Structure and Evolution of Normal Galaxies, S. M. Fall \& D. Lynden-Bell, (Cambridge: Cambridge University Press), 111

Tout, C. A. 1996, these proceedings

Zahn, J. P. 1987, in The Internal Solar angular Velocity, B. R. Durney \& S. Sofia, (Dordrecht: Reidel), 201

\section{Discussion}

M. Begelman: Models for dwarf nova outbursts required $\alpha$ to be sensitive to temperature. Do you think that magnetic viscosity models could exhibit the right kind of dependence to account for the outbursts?

S. Balbus: Yes, indications are that the level at which $\alpha$ saturates may depend upon the magnetic Prandtl number (ratio of viscosity to resistivity). Where this is high, reconnection is less efficient and $\alpha$ is bigger. In turn, the Prandtl number is very temperature sensitive; it goes like $T^{4}$, if Spitzer values are used. This is an as yet unexplored possibility.

S. Chakrabarti: To answer the question asked just now, the flow will be subKeplerian and deviate from a Shakura-Sunyaev disk no matter what the viscosity. This is because Black hole accretion is transonic.

J.-P. Lasota: What is the minimum ionisation fraction required for the BalbusHawley instability to work? Would it work for proto-stellar discs?

S. Balbus: Certainly for the classical Hayashi solar nebula models, with ionisation fractions as low as $10^{-20}$, we wouldn't expect significant magnetic coupling. On the other hand, one does not need very much ionisation for good coupling. When Omer Blaes and I looked at this in detail, we found that under a wide variety of circumstances, an ionisation fraction as low as $10^{-9}$ will do.

M. Livio: I want to point out that in a work with Armitage and Pringle we showed that you can get dwarf nova outbursts just from the fact that in the cool state the condition for the Balbus-Hawley instability is not satisfied.

S. Balbus: Provided you have a sufficiently sensitive trigger mechanism, and that you have some other way to get an $\alpha$ in the cool state, this sounds interesting. The encouraging thing to me at this point is that we're able to do something more beyond phenomenology, and that specific physical mechanisms can be discussed and simulated.

M. Romanova: This instability leads to increase of the $\alpha$-parameter to $\alpha \sim 0.1$. This means that we will have "slow" accretion like in the $\alpha$-model of Shakura \& Sunyaev. Is it correct? 
S. Balbus: The value of $\alpha$ that you cite is in fact near the upper end of what current simulations find. Whether it is "slow" or not depends entirely on context. But your question presumes that we understand and have thoroughly explored the physics of the saturation mechanisms of the instability, and this is currently not the case.

$X$. Zhang: I just wanted to make a comment that I have a poster at this conference which presents a purely gravitational viscous mechanism which is capable of producing an effective $\alpha$ in the range of $0.01-0.05$ in disks which contain large-scale non-axisymmetric instabilities.

S. Balbus: The most natural home for this sort of process is a proto-stellar disk, where low ionisation and self-gravity may tip the balance in favour of gravitational mechanisms. 